\title{
A Non-invasive, Real-Time Method for Measuring Variable Stiffness
}

\author{
Giorgio Grioli \\ Centro Interdipartimentale \\ "E. Piaggio" \\ Università di Pisa \\ Pisa, Italy 56125 \\ Email: g.grioli@centropiaggio.unipi.it
}

\author{
Antonio Bicchi \\ Centro Interdipartimentale \\ "E. Piaggio" \\ Università di Pisa \\ Pisa, Italy 56125 \\ Email: bicchi@centropiaggio.unipi.it
}

\begin{abstract}
The need for adaptability to the environment, energy conservation, and safety during physical interaction with humans of many advanced robotic applications has prompted the development of a number of Variable Stiffness Actuators (VSA). These have been implemented in a variety of ways, using different transduction technologies (electromechanical, pneumatic, hydraulic, but also piezoelectric, active polymeric, etc. ) and arrangements with elastic elements. All designs share a fundamentally unavoidable nonlinear behavior. The control schemes proposed for these actuators typically aim at independently controlling the position (or force) of the link, and its stiffness with respect to external disturbances. Although effective feedback control schemes using position and force sensors are commonplace in robotics, control of stiffness is at present completely open-loop. In practice, instead of measuring stiffness, it is inferred from the mathematical model of the actuator. Being this in most cases only roughly known, model mismatches affect severely stiffness control, undermining its utility. It should be noticed that, while for constant stiffness elements an accurate calibration of the model is possible, the same approach is hardly viable for variable stiffness systems.

We propose a method for estimating stiffness while it is varied, either intentionally or not, hence without knowledge of the command inputs. The method uses instantaneous measurements of force and position at one of the ends of the compliant elements in the system, and derives a measure that asymptotically converges to the current value of stiffness, up to an error which can be bounded by an arbitrarily small value. Simulation and experimental results are provided, which illustrate the performance of the proposed measurement method.
\end{abstract}

\section{INTRODUCTION}

To fully characterize the motion of human limbs, not only knowledge of their position and velocity, but also of their physical behavior in interaction with the environment, i.e. their mechanical impedance is needed [1, 2, 3]. Analogously, many modern robots are capable of changing their mechanical impedance to better perform a task and adapt to an environment. Variable impedance is obtained in these system by either intrinsic physical properties of the actuators (e.g. muscles in humans, or Variable Stiffness Actuators [4] in robots) or by low-level control (neural reflexes or impedance control).

Because of its ubiquitous importance, accurate measurements of the mechanical impedance of limbs are very important. Unfortunately, impedance is a rather elusive object to measure, as it is not, strictly speaking, a physical quantity (a) robotic application

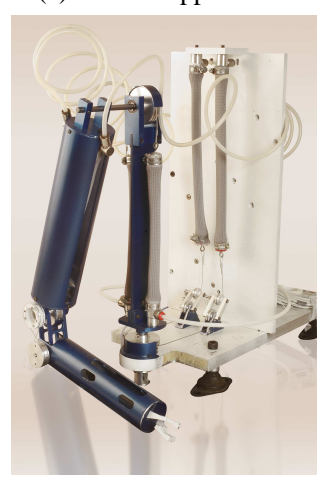

(b) biometric application

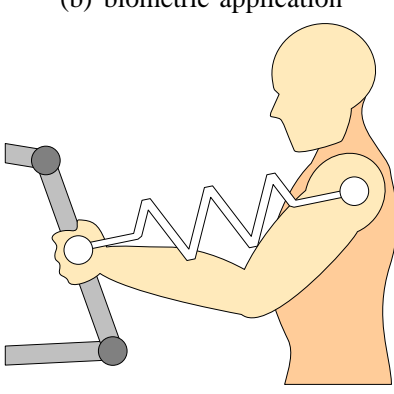

Fig. 1. Two possible applications for Non-invasive real-time stiffness measurments: control of a Variable Impedance Actuated robot (a) and identification of human limb impedance (b) [6].

per se (where by physical quantity it is meant "a property of a phenomenon, body, or substance, where the property has a magnitude that can be expressed as a number and a reference" [5]. Indeed, impedance is rather a differential operator relating the time course of physical quantities (forces and displacements). In full generality, therefore, the process of characterizing impedance of a system is more a problem of dynamical system identification than a direct measurement in a traditional sense.

Current protocols for identifying impedance in human motion typically measure the basic parameters of mass, damping, and stiffness, which concur in forming impedance, by experiments in which perturbations are purposefully injected in the system, and their effects are measured. These experiments are designed specifically so as to isolate the effects of different parameters of impedance, while at the same time minimizing perturbation of the task during which the measurement is needed [3]. In artificial robotic systems, impedance parameters are either calculated on the basis of a precise description of the model (wherever this is available), or obtained through accurate calibration procedures.

In both natural and artificial systems (e.g. see fig.1), it would be of great utility to have a method which could measure 
all impedance parameters in real time, without perturbing the normal execution of the task, and robust to inaccurate modeling and time-varying parameters which could alter calibration results. Of particular interest in robotics would be the application of such methods to the class of Variable Impedance Actuators (VIA), which have been recently introduced to address the need for adaptability to the environment, energy conservation, and safety during physical interaction with humans. These have been implemented in a variety of ways, using different transduction technologies (electromechanical, pneumatic, hydraulic, but also piezoelectric, active polymeric, etc. ) and arrangements. All designs share a fundamentally unavoidable nonlinear behavior.

The control schemes proposed for these actuators typically aim at independently controlling the position (or force) of the link, and its impedance (most often just stiffness) with respect to external disturbances. Because of the lack of a realtime technique to measure impedance, or even just stiffness, controllers proposed so far (see e.g. [7]), are in reality openloop, thus completely prone to model uncertainties.

Several works in the field of Admittance/Impedance robot control and haptic telemanipulation discuss the estimation of impedance. Online estimation of the impedance parameters of the contact dynamics is used to improve stability and overall performance of interaction and telemanipulation tasks. Most of these works assume a linear model for the environment dynamics (a simple spring, a spring-damper, or a mass-springdamper system), and use methods such as adaptive control, recursive least squares, Extended Kalman filters and Active Observers to obtain an estimate of the parameters which fits best the assumed model best with the observed measurements. An extensive list of references about those methods can be found in [11]. Other works assume nonlinear impedance contact model, as Hunt-Crossley's, see in example [12].

Our work while drawing some inspiration from those aforementioned approaches, moves a step forward with the substantial improvement of considering the possibility of dealing with a time-varying non-linear spring, without assuming any a-priori description of the spring model. Notice that nonlinear and time-varying impedance parameter are ubiquitous and unavoidable in VIA.

Moreover, in this paper, we consider the problem of realtime impedance identification along motions that are not intentionally perturbed. After setting up and discussing the problem in general terms, we focus on stiffness identification, and propose a method that uses position and force sensors to provide a measurement without recurring to any a priori knowledge of the mechanical model of the system. Simulation and experimental results are provided that confirm the viability and practical applicability of our theoretical results.

\section{Problem Statement}

The simplest example of mechanical impedance is a spring, i.e. a sample of material which exhibits a relation between the applied force $f$ and the steady-state displacement $y$ given by

$$
f=f(y) \text {. }
$$

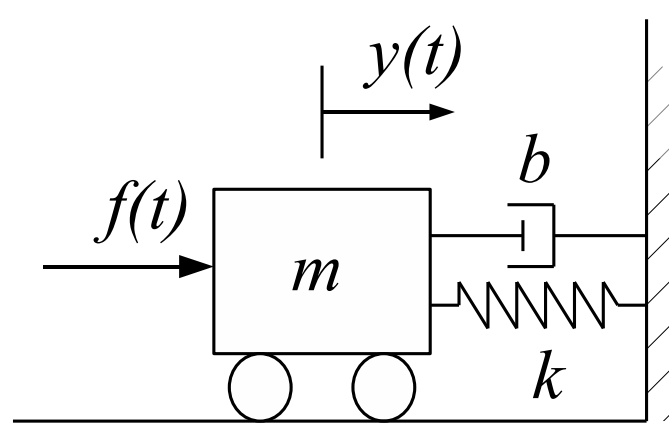

Fig. 2. A simple mass-spring-damper system.

In linear springs, $f(y)=k y$, where the constant $k$ is the spring stiffness. In general, the force function $f$ is nonlinear and time-varying, as it may depend on other parameters $u$ (which represent internal states or inputs), which in turn may vary in time. Stiffness $\sigma$ is in this case defined as

$$
\sigma(y, u)=-\frac{\partial f(y, u)}{\partial y}
$$

To introduce impedance, consider first the paradigmatic example of a mass-spring-damper system (see fig. 2), described as a relation between the applied force $f(t)$ and position $y(t)$ through

$$
f=m \ddot{y}+b \dot{y}+k y \text {. }
$$

the three parameters $m, b, k$ are constant, the O.D.E. (1) is linear and time-invariant. Introducing the Laplace transforms $F(s), Y(s)$ of force and position, one has immediately

$$
F(s)=\left(m s^{2}+b s+k\right) Y(s) .
$$

The operator $Z(s):=\left(m s^{2}+b s+k\right)$ is called the mechanical impedance of the spring-damper-mass system ${ }^{1}$. The reciprocal operator of impedance, called admittance $A(s)$, generalizes compliance as it maps forces in displacements: $Y(s)=A(s) F(s)$. The admittance operator is causal (while impedance is not).

The above approach can be generalized to a nonlinear dynamic setting by considering the relation between forces, displacements, first and second derivatives of displacements, and internal states $u$, and its graph $G \subset F \times Y \times D Y \times D^{2} Y \times$ $U$, comprised of 5-tuples $d(t):=(f(t), y(t), \dot{y}(t), \ddot{y}(t), u)$ corresponding to an idealized, infinite set of experiments. If $G(f, y, \dot{y}, \ddot{y}, u)=0$ is an analytical description of the graph, and $d_{0}$ is a regular point, then a force function $f(y, \dot{y}, \ddot{y}, u)$ is defined in a neighborhood of $d_{0}$. Defining generalized stiffness as

$$
k(d)=-\left(\frac{\partial G(d)}{\partial f}\right)^{-1} \frac{\partial G(d)}{\partial y}
$$

\footnotetext{
${ }^{1}$ It should be noted that in the literature, the term impedance is sometimes used to denote the relationship between velocity and force.
} 
generalized damping as

$$
b(d)=-\left(\frac{\partial G(d)}{\partial f}\right)^{-1} \frac{\partial G(d)}{\partial \dot{y}},
$$

generalized mass as

$$
m(d)=-\left(\frac{\partial G(d)}{\partial f}\right)^{-1} \frac{\partial G(d)}{\partial \ddot{y}}
$$

and generalized I/O characteristic as

$$
\nu(d)=-\left(\frac{\partial G(d)}{\partial f}\right)^{-1} \frac{\partial G(d)}{\partial u},
$$

one can compute the Frèchet differential of the force function as

$$
\delta f=m(d) \delta \ddot{y}+b(d) \delta \dot{y}+k(d) \delta y+\nu(d) \delta u .
$$

From consideration of the positive definiteness of the kinetic energy, Rayleigh dissipation function, and elastic energy associated with the generalized inertia, damping and stiffness, it follows that $m, b$, and $k$ are always greater than zero.

Alternatively, one can describe admittance along a given trajectory as the linear operator mapping small changes of the external force to changes in the resulting motion. To do this, consider the nonlinear ODE obtained by solving $G(f, y, \dot{y}, \ddot{y}, u, t)=0$ at a regular point with respect to $\ddot{y}$ as

$$
\ddot{y}=g(y, \dot{y}, f, u)
$$

and its state space form, with $x \in \mathbb{R}^{2}, x_{1}=y, x_{2}=\dot{y}$, i.e.

$$
\frac{d}{d t}\left[\begin{array}{l}
x_{1} \\
x_{2}
\end{array}\right]=\left[\begin{array}{c}
x_{2} \\
g\left(x_{1}, x_{2}, f, u\right)
\end{array}\right]
$$

For given initial conditions $x(0)=\bar{x}_{0}$ and a given course in time for force $\bar{f}(t)$, let $\bar{x}(t)$ be the trajectory obtained, i.e. the solution of the dynamics ODE. The first-order approximation of the dynamics of the perturbed motion $\tilde{x}(t)=x(t)-\bar{x}(t)$ corresponding to a change in force $\tilde{f}(t)=f(t)-\bar{f}(t)$ is the time-varying linear system

$$
\dot{\tilde{x}}=\Gamma(t) \tilde{x}+\Theta(t)\left[\begin{array}{l}
\tilde{f} \\
\tilde{u}
\end{array}\right]
$$

where

$$
\Gamma(t)=\left[\begin{array}{cc}
0 & 1 \\
-\frac{k(d)}{m(d)} & -\frac{b(d)}{m(d)}
\end{array}\right], \quad \Theta(t)=\left[\begin{array}{cc}
0 & 0 \\
\frac{1}{m(d)} & \frac{\nu(d)}{m(d)}
\end{array}\right] .
$$

Example: Consider the link in fig. 3, actuated by two antagonistic actuators (with a role vaguely similar to that of the biceps and triceps muscles at the elbow), with a quadratic damping and subject to gravity and to an external torque load $\tau_{e}$. The system dynamics are

$$
I \ddot{\theta}+\beta \dot{\theta}|\dot{\theta}|-\tau_{b}+\tau_{t}-m g l \sin \theta-\tau_{e}=0
$$

Assume first that the two actuators generate torques according to the model (cf. [8])

$$
\begin{aligned}
& \tau_{b}=\left(\tau_{\max }-\alpha \theta_{b}\right) u_{b} \\
& \tau_{t}=\left(\tau_{\max }-\alpha \theta_{t}\right) u_{t}
\end{aligned}
$$
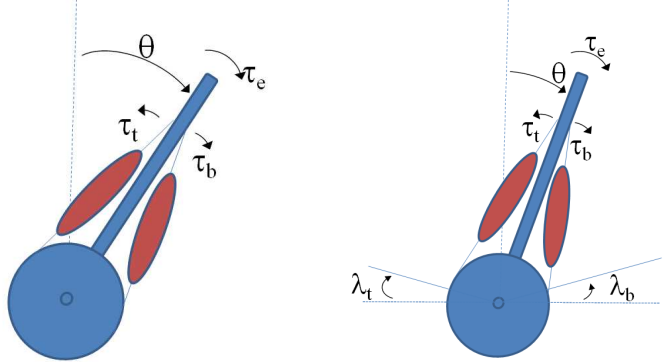

Fig. 3. A link subject to an external load and actuated by two antagonistic actuators. Nomenclature refers to actuator models used in (3) and (4), respectively.
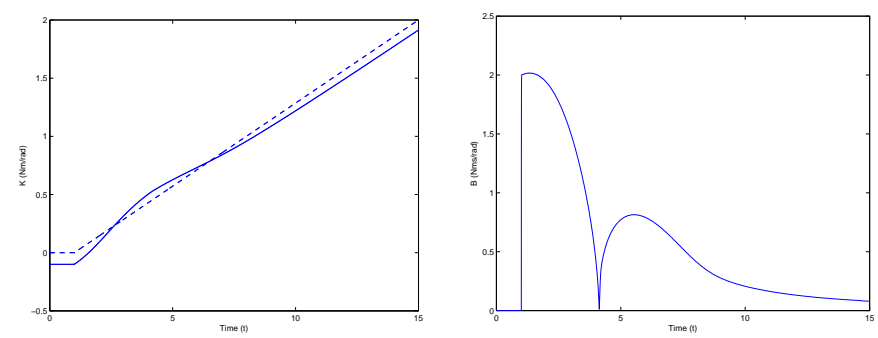

Fig. 4. Generalized stiffness (left - dashed is without gravity term) and generalized damping for the example with actuators as in (3), subject to a unit step in external torque at $T=1 \mathrm{~s}$, and with time-varying activation $u_{b}(t)=u_{t}(t)$ linearly increasing from 0 at $T=1 \mathrm{~s}$ to 1 at $T=15 \mathrm{~s}$. Numerical values used in simulation: $I=0.05 \mathrm{Nms}^{2}, m g l=0.1 \mathrm{Nm}, \beta=$ $1 \mathrm{Ns}^{2} \mathrm{~m}$, alpha $=1 \mathrm{Nm} / \mathrm{rad}$, tau $_{\max }=2 \mathrm{Nm}$.

where $\theta_{b}=(\pi / 2+\theta), \theta_{t}=(\pi / 2-\theta), \tau_{\max }$ is the maximum isometric torque, $u_{b}, u_{t}$ are the normalized contraction parameters $\left(0 \leq u \leq 1, u=u_{b}, u_{t}\right)$, and $\alpha$ is a constant assumed to be equal for the two actuators. We easily obtain $m=I$ for the generalized mass, $b(\dot{\theta})=2 \beta|\dot{\theta}|$ for the generalized damping, and

$$
k(\theta, u)=\alpha\left(u_{b}+u_{t}\right)-m g l \cos (\theta)
$$

for the generalized stiffness. In the latter expression, the role of a gravity-induced term and a co-activation stiffness term are apparent.

If a different actuator model is adopted, namely (cf. [9])

$$
\begin{aligned}
& \tau_{b}=-\alpha\left(\theta_{b}-\lambda_{b}\right)^{2}, \\
& \tau_{t}=-\alpha\left(\theta_{t}-\lambda_{t}\right)^{2}
\end{aligned}
$$

where $\lambda_{b}, \lambda_{t}$ are interpreted as the rest lengths of the actuators, one has

$$
k(\theta)=2 \alpha\left(\pi-\lambda_{b}-\lambda_{t}\right)-m g l \cos (\theta)
$$

The values of stiffness and damping for the two examples above, corresponding to time-varying values of the control parameters, are reported in figs. 4 and 5, respectively.

\section{IMPEDANCE OBSERVERS}

For the motivations above introduced, we seek a method for characterizing impedance using force and position measurements taken during the natural evolution of a system, as 

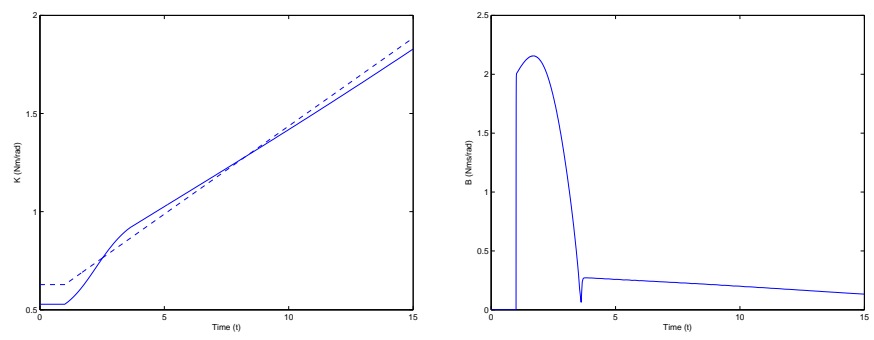

Fig. 5. Generalized stiffness (left - dashed is without gravity term) and generalized damping for the example with actuators as in (4), subject to a unit step in external torque at $T=1 \mathrm{~s}$, and with time-varying reference angle $\lambda_{b}(t)=\lambda_{t}(t)$ linearly decreasing from $\pi / 3$ at $T=1 \mathrm{~s}$ to 0 at $T=15 \mathrm{~s}$. Numerical values used in simulation as in fig. 4 , except for $\alpha=0.3$.

e.g. represented by the reference motion $\bar{x}(t)$ under a given reference excitation $\bar{f}(t)$, assuming that the design of the excitation $\bar{f}(t)$ is not at our disposal.

According to the description of impedance as a differential operator, its characterization can be cast as an identification problem of a dynamical system. This is done first for the case of linear impedance as follows.

\section{A. Observers for linear impedance}

Consider an extended state vector $z=$ $\left[\begin{array}{lllll}y & \dot{y} & -\frac{k}{m} & -\frac{b}{m} & \frac{1}{m}\end{array}\right]$, and rewrite the dynamics of (1) as

$$
\begin{aligned}
\dot{z} & =\left[\begin{array}{c}
z_{2} \\
z_{1} z_{3}+z_{2} z_{4} \\
0 \\
0 \\
0 \\
y
\end{array}\right]+\left[\begin{array}{c}
0 \\
z_{5} \\
0 \\
0 \\
0
\end{array}\right] f \\
& =z_{1}
\end{aligned}
$$

The identification of the impedance parameters can thus be cast as a nonlinear state estimation problem, i.e., from the measurement of the external force $f$ and position $y$, estimate the initial state $z(0)$, and in particular its three last components which completely determine the linear impedance.

We preliminarily establish that the problem is well posed. Indeed, considering the observability codistribution for this system,

$$
\Omega(z)=\left[\begin{array}{ccccc}
1 & 0 & 0 & 0 & 0 \\
0 & 1 & 0 & 0 & 0 \\
z_{3} & z_{4} & z_{1} & z_{2} & 0 \\
0 & 0 & 0 & 0 & 1 \\
z_{3} z_{4} & z_{3}+z_{4}^{2} & z_{2}+z_{1} z_{4} & z_{1} z_{3}+2 z_{2} z_{4} & 0 \\
0 & 0 & 0 & z_{5} & z_{4}
\end{array}\right]
$$

it turns out that for $m, b, k>0$, $\operatorname{dim} \Omega(z)^{\perp}=0, \forall z$ except $z_{1}=z_{2}=0$. Hence, if the system moves from the equilibrium, the three linear impedance parameters can be reconstructed from position and force measurements.

To actually estimate the impedance in this case, different methods can be adopted. These include standard off-line identification techniques (which exploit the linear nature of the regressor for the unknown parameters), such as e.g. in [10], or on-line nonlinear state observers (e.g. Extended Kalman Filters) applied to system (5).

\section{B. Variable stiffness observers}

Unfortunately, generalization of the above straightforward approach to the case when impedance is nonlinear and/or time varying is not trivial. To convince oneself, it is sufficient to consider the case of an unknown force function $s(y, u(t))$ replacing the linear spring term in (1), i.e.

$$
f=m \ddot{y}+b \dot{y}+s(y, u),
$$

when no information on the structure of $f$ or on the variable $u(t)$ is available ${ }^{2}$.

In the rest of this paper we describe a different approach to measure stiffness in a system such as (6). For simplicity's sake, let us assume for the time being that accurate measures of the applied force $f(t)$ and of the position $y(t)$ are available, and that numerical derivatives of these signals can be done. Assume also that both the mass and damping coefficients, $m$ and $b$, are known (these strong assumptions will be discussed later on). No assumptions are made on the function $s(y, u)$ except that it is smooth in both arguments, with bounded derivatives of all orders.

We assume that the stiffness-regulating input $u(t)$ is bounded with its first derivative $\dot{u}(t)$. It should be noticed that, in building an observer that relies only on measurements of the position $y(t)$ corresponding to the external load $f(t)$, it is physically impossible to observe a stiffness which is changing in time $(\dot{u}(t) \neq 0)$ while the system is at equilibrium $(\dot{y}(t)=0)$. More precisely then, we will make the assumption that the ratio between the stiffness regulation rate of change and the velocity of the measured trajectory is bounded, namely that, for all times $t$ during the application of the observer, it holds

$$
\frac{|\dot{u}(t)|}{|\dot{y}(t)|}<v \in \mathbb{R}, \quad \forall t .
$$

Let

$$
\frac{\partial f}{\partial y}=\frac{\partial s(y, u)}{\partial y}:=\sigma(y, u(t))
$$

denote the stiffness to be measured. Also let $\hat{\sigma}(t)$ denote its estimate at time $t$, and $\tilde{\sigma}(t)=\sigma(y, u(t))-\hat{\sigma}(t)$ be the estimation error.

Differentiate (6) once with respect to time to get

$$
\dot{f}=m \dddot{y}+b \ddot{y}+\sigma \dot{y}+s_{u} \dot{u},
$$

where $s_{u}:=\frac{\partial s(y, u)}{\partial u}$. Using the current estimate of stiffness and the assumptions stated above, a best-effort prediction for $\dot{f}$ can be written (in the absence of information on $s(y, u)$ and on $u(t))$ as

$$
\dot{\hat{f}}=m \dddot{y}+b \ddot{y}+\hat{\sigma} \dot{y}
$$

${ }^{2}$ In the case a parametric description of the force function is available, e.g. in terms of a finite polynomial expansion $f(y, u)=k_{0}(u)+k_{1}(u) y+$ $k_{2}(u) y^{2}+\ldots$, and $u$ is constant, an observable finite dimensional nonlinear system can be built. However, the possibility to achieve good performance of the corresponding observer is dubious. 
We will show that the update law

$$
\dot{\hat{\sigma}}=\alpha \dot{\tilde{f}} \operatorname{sgn}(\dot{y})
$$

with $\alpha>0$ and

$$
\operatorname{sgn}(x):=\left\{\begin{array}{cl}
\frac{x}{\|x\|} & \text { if }\|x\| \neq 0 \\
0 & \text { if }\|x\|=0
\end{array},\right.
$$

is such that $\hat{\sigma}(t)$ can be made to converge to the true stiffness value $\sigma(t)$ within an uniformly ultimately bounded error.

Indeed, consider the positive definite error function

$$
V_{\sigma}:=\frac{1}{2} \tilde{\sigma}^{2}
$$

and its derivative along the trajectory defined in (7), i.e.

$$
\dot{V}_{\sigma}=\tilde{\sigma} \dot{\tilde{\sigma}}=\tilde{\sigma} \dot{\sigma}-\tilde{\sigma} \dot{\hat{\sigma}}=\tilde{\sigma} \dot{\sigma}-\alpha \tilde{\sigma} s_{u} \dot{u} \operatorname{sgn}(\dot{y})-\alpha \tilde{\sigma}^{2}|\dot{y}| \text {. }
$$

While the first two terms in the rightmost sum in (8) are indefinite in sign, the third term is negative definite. Therefore, wherever the inequality holds

$$
|\tilde{\sigma}|>\left|s_{u}\right| \frac{|\dot{u}|}{|\dot{y}|}+\frac{1}{\alpha} \frac{|\dot{\sigma}|}{|\dot{y}|}
$$

the derivative of the error function $\dot{V}_{\sigma}$ is negative, hence the estimation error decreases. By writing $\dot{\sigma}=\sigma_{y} \dot{y}+\sigma_{u} \dot{u}$, and using the upper bound above assumed on the rate of stiffness change, we have that stiffness estimates converge to the true value within an ultimately uniformly bounded error given by

$$
|\tilde{\sigma}|>\frac{\left|\sigma_{y}\right|}{\alpha}+\left(\left|s_{u}\right|+\frac{\left|\sigma_{u}\right|}{\alpha}\right) v
$$

Remark: The assumption that the mass and damping are exactly known is not realistic. However, it is easy to verify that the analysis above carries over exactly even with no knowledge of $m$ and $b$, provided that a force sensor directly placed on the elastic element provides a measure of the force $s(x, u)$. In case this is not available, then errors on the $m$ and $b$ parameters have the effect of making the ultimate error larger (this effect can be countered by increasing the observer gain $\alpha$ ).

\section{Simulation Results}

As a first illustration, the proposed stiffness observer is tested using numeric simulations in two systems.

\section{A. Single spring}

In this first test, the observer algorithm is used to track the stiffness value of a single spring with non-linear, time-invariant stiffness characteristic. The simulated elastic element is an exponential spring, whose force/displacement characteristic is

$$
s(y)=a e^{b y} .
$$

Such springs are designed so that the stiffness at any operating point is proportional to the force the spring is exchanging with the external environment.

Figure 5(a) compares the observer estimate of the spring stiffness with its exact value $\sigma(y)=b a e^{b y}$, for three different values of the spring parameters. The springs were subject to a external force $f(t)$ given by a chirp in the frequency range from 0.1 to $10 \mathrm{~Hz}$, with deformations $y(t)$ in the range $[0.25,2.25] \mathrm{cm}$. The observation error (shown in fig. 5(b)) decays to less than one percent in less than $0.3 \mathrm{~s}$.

(a) tracking
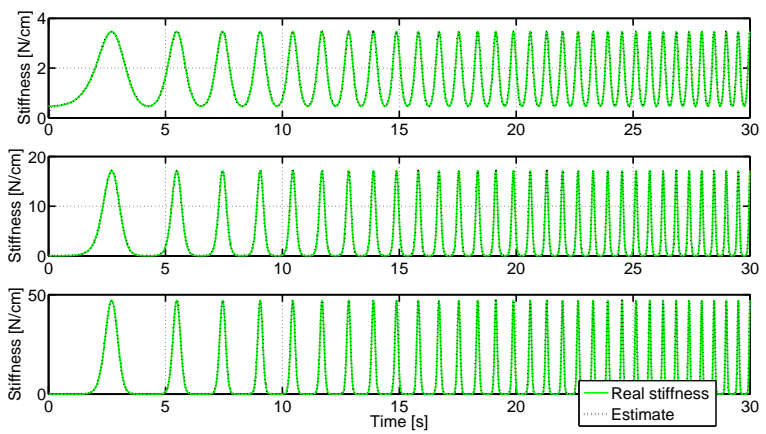

(b) error
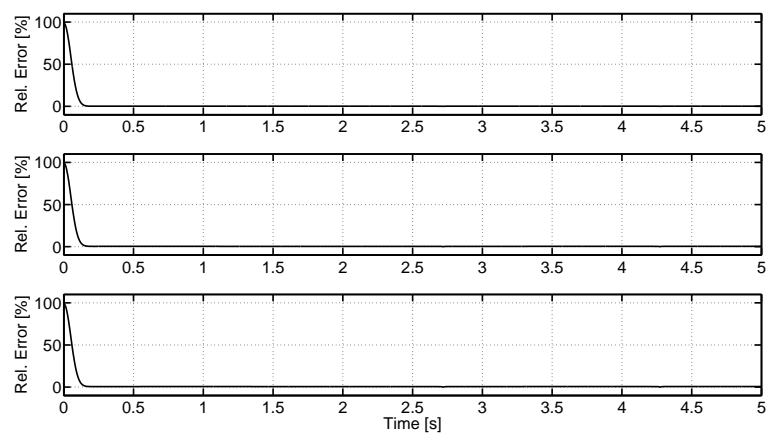

Fig. 6. Simulation results for the exponential spring test, with three different springs $\left(a=2.7 e^{-2}, b=1 ; a=8.1 e^{-6}, b=3 ; a=13.5 e^{-10}, b=5\right.$, respectively). Stiffness tracking is shown in panel (a), and relative estimation error in panel (b). Only the first 5 s of the relative error are shown, to focus on the transient phase. Relative error remains under $1 \%$ for the rest of the time.

\section{B. Antagonistic VSA systems}

One of the simplest and most common examples of variable stiffness, both in natural systems and in robotics, is the agonistantagonist arrangement on nonlinear actuators. To illustrate how our proposed stiffness observer applies to antagonist VSA systems, consider the examples reported above in fig. 3 . Application of the stiffness observer in this case can be carried out in two ways: 1) the tendon tensions $\tau_{b}, \tau_{t}$ are measured directly, or 2) the external torque $\tau_{e}(t)$ is measured, and estimates of the link inertia and damping are used. In all cases, a measurement of the link angle $\theta(t)$ is necessary. It should be noticed that, while the first method does not require any estimate of link parameters, it is more invasive in the system, and is inapplicable to e.g. stiffness measurement in a human elbow joint. On the opposite, the second method is easily applicable to this case, although its accuracy will be reduced if poor estimates of inertia and damping are available.

Simulation results for the antagonist arrangements of two muscle-like actuators as described in (3) and (4) are reported in fig. 7 a) and b), respectively. In both simulations, the external 
(a) example 1

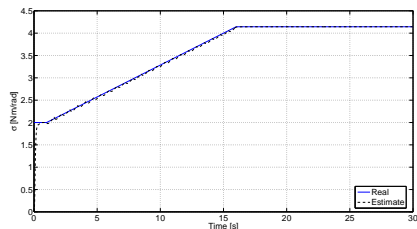

(b) example 2

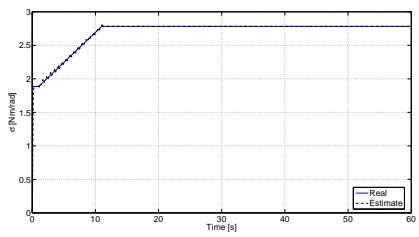

Fig. 7. Stiffness tracking for an antagonist VSA system realized adopting nonlinear muscle-like actuators as in equations 3 and 4 (panels (a) and (b) respectively). (a) example 1

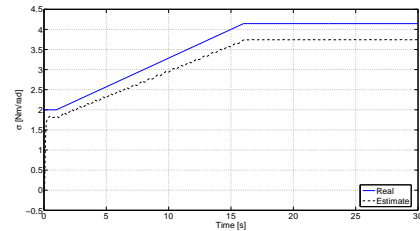

(b) example 2

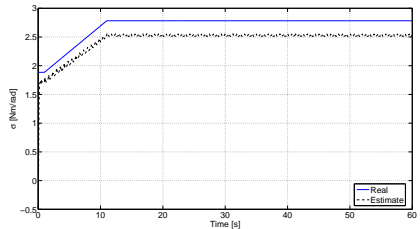

Fig. 8. Stiffness tracking of the antagonist VSA systems with muscle-like actuators as in (3) (a), and (4) (b), with a $10 \%$ error in the knowledge of parameters $m$ and $b$.

force $\tau_{e}$ is a sinusoid with $\omega=5 \mathrm{rad} / \mathrm{s}$ and amplitude 0.02 $\mathrm{Nm}$. Stiffness is varied during the simulation in a saturated ramp fashion. The ensuing joint motion $\theta(t)$ is in the range $\pm 0.01 \mathrm{rad}$, while $\dot{\theta}$ varies from zero to $0.05 \mathrm{rad} / \mathrm{s}$.

To assess how strongly the performance of the stiffness observer is affected by inertia and damping parameter mismatches (in the case that only external torques are measured), we performed simulations in the hypothesis that $m$ and $b$ were in error by $10 \%$ of their actual value. Results reported in fig. 8 indicate that, for both muscle models, the relative error on stiffness is of comparable magnitude.

Finally, a simulation is reported for the same link actuated by two exponential springs (9). In this case, the stiffness is exponentially increasing in time (by linearly varying the cocontraction of the antagonist springs). A sinusoidal external force is applied during the initial and final phases of the experiment, while it is set to zero in the time interval between 10 s and 20s. Correspondingly, motion of the links stops $(\dot{\theta}=$ $0)$, and the stiffness estimate is not updated in the interval. When motion resumes, the estimation recovers quickly to the exact value.

\section{EXPERIMENTAL RESULTS}

The algorithm has been tested on the experimental device shown in figure 10, implementing the antagonistic VSA device with exponential springs described previously. Two straingauge load cells were used to measure the tendon tensions directly, while positions of the link and of the tendon origin were measured using three HEDS-5540 encoders with a resolution of 2000 CPRs. Data were acquired using a National Instruments PCI6251 ADC board for the strain gauges, and an USB-PCI4e for the encoders. Data were sampled with sampling time $T_{s}=0.015 \mathrm{~s}$, and afterward filtered with (a) tracking

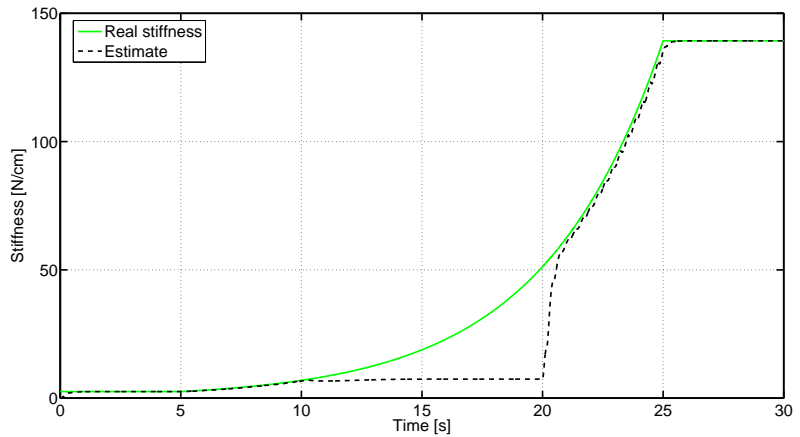

(b) relative error

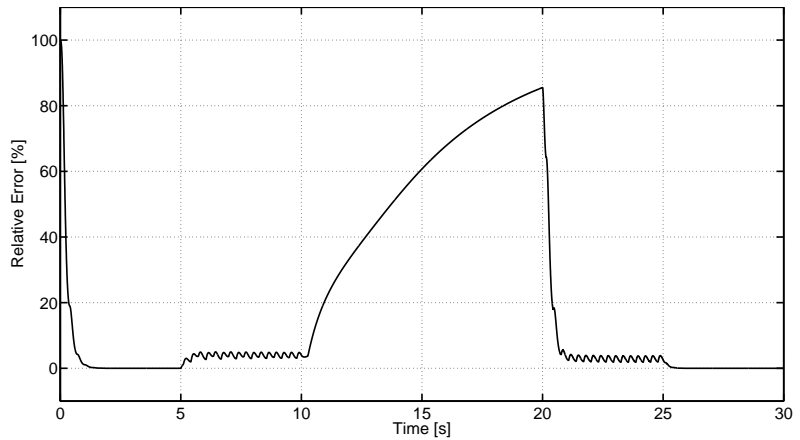

(c) link movement

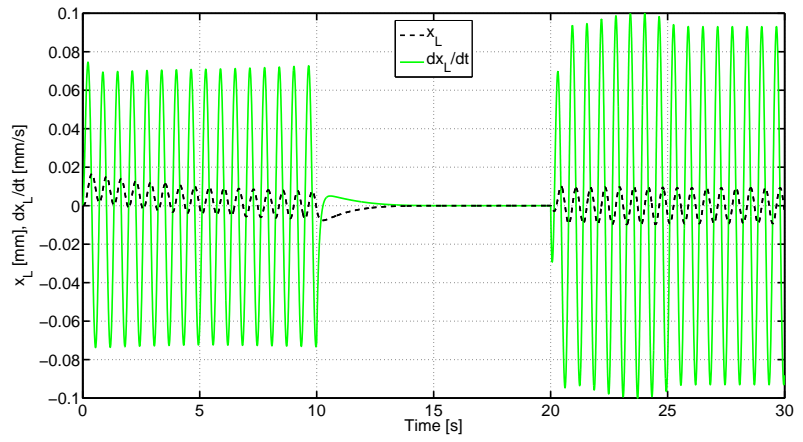

Fig. 9. Simulation results for the VSA system with exponential springs. a) Comparison of the link stiffness with its estimate; b) Relative estimation error; c) link motion during the simulated experiment.

a second-order filter with time constant of $0.02 \mathrm{~s}$. Signal derivatives used in the algorithm were approximated by the numerical filter described by the transfer function

$$
D(s)=\frac{s}{1+10^{-4} s} .
$$

Despite the simplicity of such "Dirty Derivatives" technique, results were suitable for the purposes of the present work, proving the practical feasibility of the proposed method.

It should be pointed out that in this paper the impedance estimates are not used for control in a feedback loop, hence the effect of derivation noise can not destabilize the system. Future work will address this problem when closed loop control of impedance will be considered.

Input signals derivation could be avoided through use of sensors of the rate of change of desired quantities, e.g. 


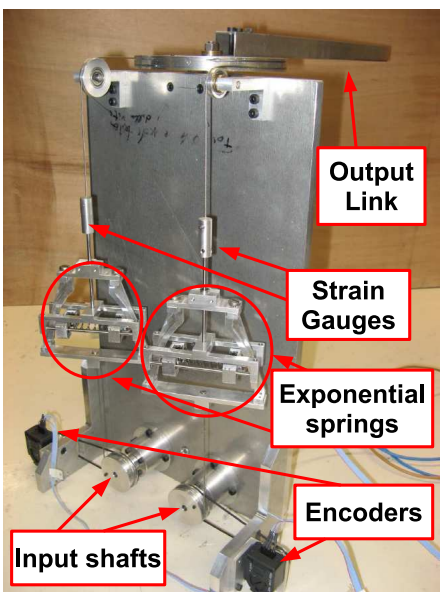

Fig. 10. The experimental setup consists of an antagonistic VSA system with exponential springs, realized using a linear spring forced to move on a suitable cam profile. Force sensors (strain gauges) are mounted on the tendons connecting the springs to the link. Position sensors (encoders) are mounted on the link and on two tendon pulleys coupled with the input levers.

inductive sensors for positions, and piezo-electric sensors for forces.

Both the external load and the torque actuating the tendon tensions were generated manually, and not measured.

To obtain ground-truth data, the force functions of the two springs were experimentally evaluated through careful preliminary calibration experiments. The calibration procedure consisted in collecting a large number of force-displacement pairs $(x, f)$, translating them in semi-logarithmic coordinates space $(x, y=\ln (f))$, finding the regression line in the semilogarithmic space, such that $y=m x+q$, to finally go back to the original space and obtain $f=e^{y}=e^{m x+q}=e^{q} \cdot e^{m x}$, from which $a=m$ and $b=e^{q}$. From the mean square error of the regression $S M E$, the relative error margin of the model can be easily evaluate as $r=1-e^{S M E}$.

The numeric values of the exponential curves fitting our data are

$$
\begin{array}{ll}
a_{1}=0.999, & b_{1}=3.267, \\
a_{2}=0.950, & b_{2}=2.780,
\end{array}
$$

where subscripts are relative to the two spring. Figure 11 shows the regressed curve alongside with experimental data for both the left (a) and right (b) springs. It is noticeable that, due to un-modeled friction in the mechanism implementing the exponential springs, a certain hysteresis is present, making the model correct only up to a relative error margin of about $25 \%$. Anyway, this error only marginally affected the performance of the proposed observer.

Raw experimental data are reported in fig. 12. The estimate of stiffness reconstructed in real-time by the stiffness observer $(\alpha=3)$ is compared with the calibrated stiffness data in fig. 13

\section{CONCLUSION}

In this paper we presented an algorithm which can be used to measure stiffness in real-time, using force and position (a) left spring

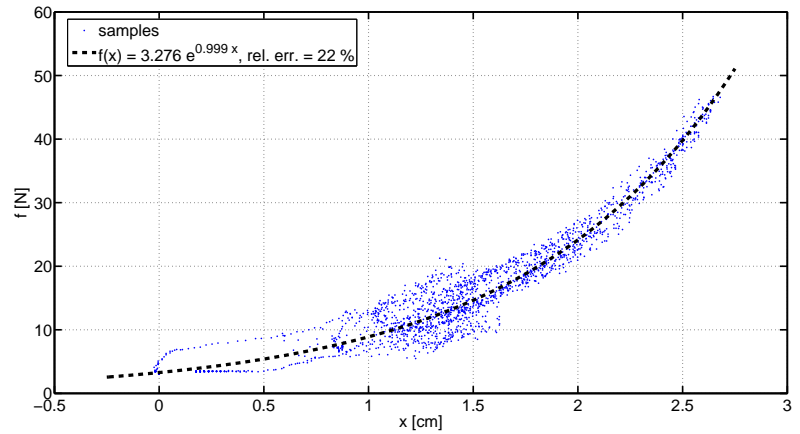

(b) right spring

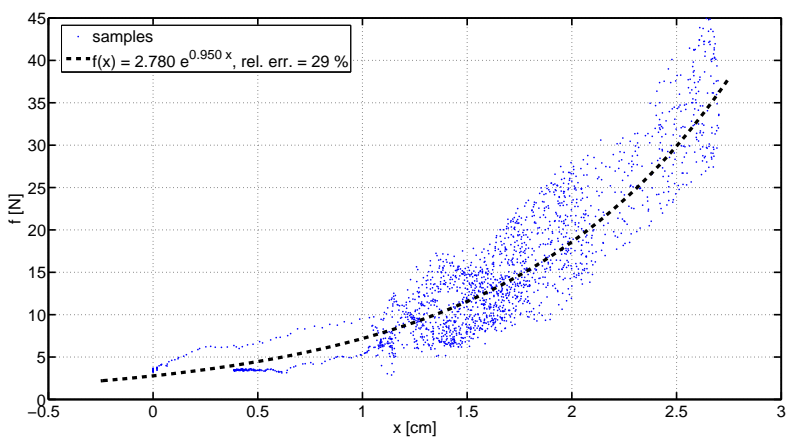

Fig. 11. Experimental characterization of the two exponential springs. Force and displacement pairs recorded during a calibration experiment, and regression curve are shown for each of the two springs of the experimental VSA system.

sensors. The method's main advantages are that it does not require injecting any intentional perturbation in the system, and that uses no a piori knowledge on the model of the physical actuators. Simulations and experimental test shown that the method is practically applicable and robust to noisy data and uncertain parameters.

\section{ACKNOWLEDGMENT}

The authors wish to thank Alessandro De Luca and Riccardo Schiavi for the useful discussions, and Soumen Sen for the help on the realization of the experimental set-up. Further gratitude goes to the anonymous reviewers for their mindful comments and constructive critiques. This work was partially supported by the VIACTORS and THE projects, funded by the European Community under contract IST - 2315542008 and ICT - 248587 - 2010, respectively.

\section{REFERENCES}

[1] N. Hogan, "Impedance control: An approach to manipulation, part i - theory, part ii - implementation, part iii - applications," Journal of Dynamic Systems, Measurement and Control, vol. 107, pp. 1-24, 1985.

[2] M. Kawato, "Internal models for motor control and trajectory planning," in Current Opinion in Neurobiology. Elsevier Science Ltd., 1999, vol. 9, pp. 718-727.

[3] E. Burdet, R. Osu, D.W. Franklin, T. Yoshioka, T.E. Milner and M. Kawato, "A method for measuring endpoint stiffness during multijoint arm movements," Journal of Biomechanics, vol. 33, no. 12, pp. 1705-1709(5), December 2000.

[4] B. Vanderborght K.W. Hollander R. Ham, T. Sugar and D. Lefeber, "Compliant actuator designs," IEEE, Robotics and Automation Magazine, vol. 16, no. 3, pp. 81-94, September 2009. 
(a) positions

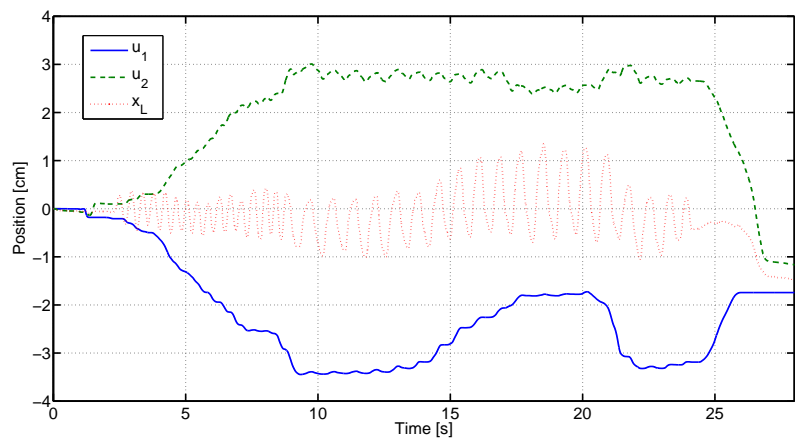

(b) forces

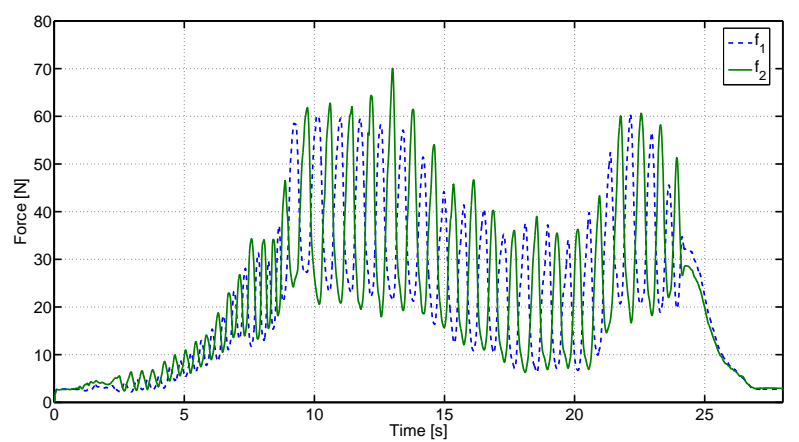

(c) observer inputs
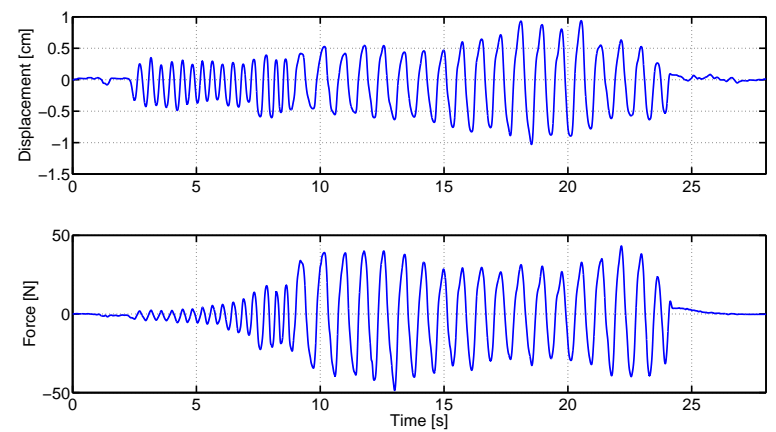

Fig. 12. Experimental data. Raw data recording from sensors is shown in the first two panels. The third panel shows the values of $f$ and $x$ actually fed to the observer during the experiment.

[5] J. C. for Guides in Metrology (JCGM), Ed., International Vocabulary of Metrology (VIM), iii ed. Pavillon de Breteuil, 2008, vol. Basic and General Concepts and Associated Terms, no. JCGM 200:2008, http://www.bipm.org/utils/common/ documents/jcom/JCGM 200_2008.pdf.

[6] B. Sepehri1, A. Esteki, E. Ebrahimi-Takamjani, G.-A. Shahidi, F. Khamseh, and M. Moinodin "Quantification of rigidity in Parkinson disease," inAnnals of Biomedical Eng., vol. 35, no. 12, pp. 2196-2203, December 2007. (a) tracking

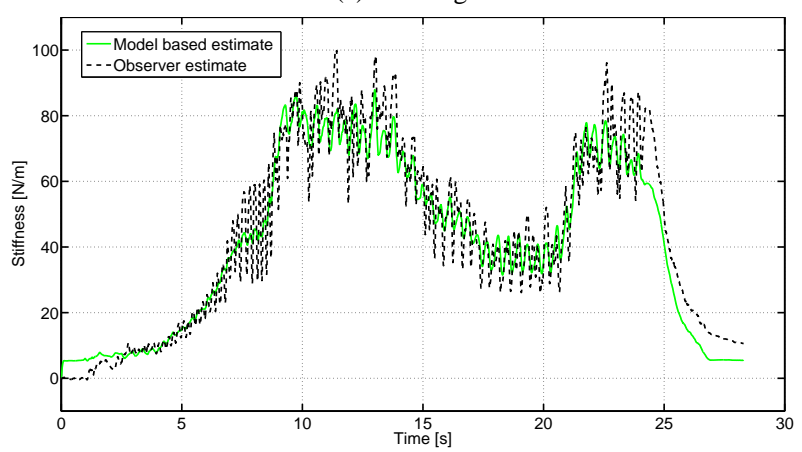

(b) relative error

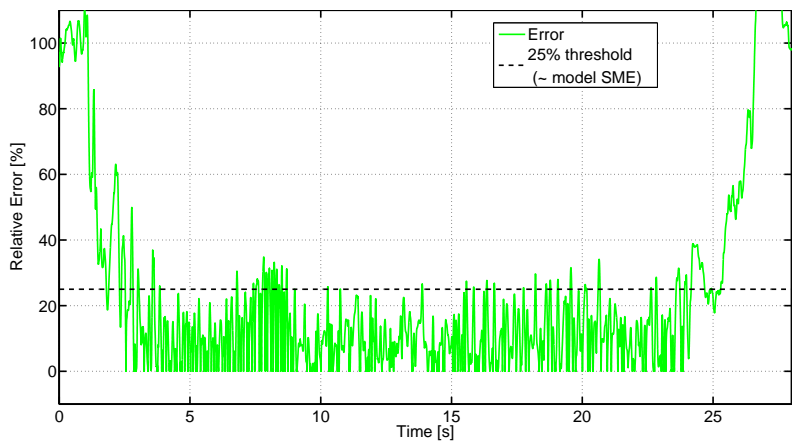

Fig. 13. Experimental results for the stiffness observer. The first panel shows a comparison between the stiffness values derived by the calibrated spring model and the estimate performed by the stiffness observer algorithm. The second panel shows the relative error (difference between estimator and model, normalized w.r.t. the model) compared with the relative error underlying the model itself.

[7] A. De Luca and P. Lucibello, "A general algorithm for dynamic feedback linearization of robots with elastic joints," in IEEE Int. Conf. on Robotics and Automation, 1998, pp. 504-510.

[8] N. Hogan, "Adaptive control of mechanical impedance by coactivation of antagonist muscles," IEEE Trans. Aut. Cont., vol. AC-29, no. 8, pp. 681-690, August 1984.

[9] A.G. Feldman, "Functional tuning of the nervous system with control of movement or maintenance of a steady posture. ii: Controllable parameters of the musc1e," Biophys., vol. 11, pp. 565-578, 1966.

[10] K. Hashimoto, T. Kureha, Y. Nishimura, K. Okumura, and S. Muraoka, "Measurement of mechanical impedance using quartz resonator force sensor during the process of grasping," in SICE 2004 Annual Conference, vol. 1, pp. 722-726, August 2004.

[11] D. Verscheure, I. Sharf, H. Bruyninckx, J. Swevers, and J. De Schutter, "Identification of Contact Dynamics Parameters for Stiff Robotic Payloads," in IEEE Trans. Robot., vol. 25, no. 2, pp. 240-252, April 2009.

[12] N. Diolaiti, C. Melchiorri, and S. Stramigioli, "Contact impedance estimation for robotic systems," in IEEE Trans. Robot., vol. 21, no. 5, pp. 925-935, October 2005. 